\title{
Contributions of De Novo Synthesis of Fatty Acids to Total VLDL-Triglyceride Secretion during Prolonged Hyperglycemia/Hyperinsulinemia in Normal Man
}

\author{
Asle Aarsland, David Chinkes, and Robert R. Wolfe \\ Metabolism Unit, Shriners Burns Institute and University of Texas Medical Branch, Galveston, Texas 77550
}

\begin{abstract}
Triglycerides (TG) are synthesized in the liver principally from two sources of fatty acids (FA): FA synthesized de novo in the liver and preformed FA. We have measured the rate of secretion of de novo synthesized FA and total secretion of FA bound to VLDL-TG in healthy men $(n=5)$ in the basal state, and after 1 (day 1) and $4 \mathrm{~d}$ (day 4 ) of a hypercaloric carbohydrate diet $(\approx 2.5$ times energy expenditure) that generated a moderate endogenous hyperinsulinemia (plasma insulin $\approx 60 \mu \mathrm{U} / \mathrm{ml}$ ). Prolonged carbohydrate hyperalimentation/hyperinsulinemia increased plasma VLDL$\mathrm{TG} \approx 10$-fold in part due to a 3.4-fold increase in total VLDL-TG secretion rate (basal state $=72 \pm 23$, day $4=$ $242 \pm 78 \mu \mathrm{mol} \mathrm{TG} / \mathrm{kg} / \mathrm{d}$ ). Although the secretion of de novo synthesized FA increased throughout the study (basal state $=1.1 \pm 0.4$, day $1=15.9 \pm 7.9$, day $4=50.0 \pm 18.8 \mu \mathrm{mol} \mathrm{TG} /$ $\mathrm{kg} / \mathrm{d}$ ), the 2.7-fold increase in secretion rate of preformed FA (basal state $=70 \pm 23$, day $4=191 \pm 57 \mu \mathrm{mol} \mathrm{TG} / \mathrm{kg} / \mathrm{d}$ ) quantitatively contributed the most to total VLDL-TG secretion rate. Decreased catabolism of VLDL-TG also contributed to the hypertriglyceridemia as reflected by an approximately fourfold decrease in both fractional turnover rate (basal state $=9.2 \pm 3.8$, day $1=2.1 \pm 0.2$, day $4=$ $2.1 \pm 0.3$ pools $/ \mathrm{d}$ ) and rate of clearance (basal state $=$ $0.35 \pm 0.08$, day $1=0.11 \pm 0.01$, day $4=0.09 \pm 0.01$ liter $/ \mathrm{kg} /$ d) of VLDL-TG. Thus, the primary difference between 1 and $4 \mathrm{~d}$ of hyperinsulinemia in conjunction with carbohydrate hyperalimentation is the increase in hepatic secretion of preformed FA into VLDL-TG. (J. Clin. Invest. 1996. 98: 2008-2017.) Key words: triglycerides • liver • carbohydrate • hyperalimentation $\bullet$ stable isotopes
\end{abstract}

\section{Introduction}

The metabolic link between insulin resistance and the associated risk factors for atherosclerotic cardiovascular disease, especially dyslipidemia, has been the focus of intensive investigation in recent years. Of the several abnormalities in lipoprotein metabolism associated with insulin resistance, hypertriglyceridemia is the most common (1-3) and of particular interest and significance because of the growing awareness that hypertriglyceridemia itself may be important in atherogenesis (4-6).

Address correspondence to Robert R. Wolfe, Shriners Burns Institute, 815 Market Street, Galveston, TX 77551. Phone: 409-770-6605; FAX: 409-770-6825.

Received for publication 15 September 1995 and accepted in revised form 15 August 1996.

J. Clin. Invest.

(C) The American Society for Clinical Investigation, Inc. 0021-9738/96/11/2008/10 \$2.00

Volume 98, Number 9, November 1996, 2008-2017
Whereas it is generally agreed that hypertriglyceridemia in insulin-resistant states results from a combination of an overproduction of VLDL particles in the liver and a decreased catabolism of triglycerides (TGs) ${ }^{1}$ peripherally $(2,7,8)$, the exact cause of the abnormality remains controversial. Although hyperinsulinemia has been commonly thought of as a promoter of TG production $(9,10)$, no causal relationship has been found between hyperinsulinemia and hypertriglyceridemia in human subjects. Thus, while studies using perfused rat livers have generally shown an acute stimulatory effect of insulin (11), experimentally induced acute hyperinsulinemia suppresses VLDL-TG production in human subjects (12). Under most conditions, hepatic TGs are predominantly produced from the reesterification of FAs cleared from the plasma, and consequently the delivery of FFA to the liver has often been cited as the most important factor controlling VLDL-TG secretion (13). This strong relationship between lipolysis and VLDL-TG production makes a stimulatory effect of insulin on VLDL-TG production unlikely, because insulin is a potent inhibitor of lipolysis (14). Thus, peripheral resistance to the antilipolytic effect of insulin may be necessary for a direct stimulatory effect of insulin on VLDL-TG secretion to be observed in vivo (15). Alternatively, it is possible that insulin stimulates VLDL-TG output by stimulating de novo FA synthesis (16). However, recent data directly quantifying the contribution of newly synthesized FAs to VLDL-TG secretion during high carbohydrate ingestion give reason to question the physiological importance of this source of FAs in the production of VLDL-TG (17). Finally, it is possible that insulin does not play a direct role in the regulation of hepatic VLDL-TG production and that VLDL-TG production is increased in insulin-resistant states because of hyperglycemia. This notion is supported by the observation that the infusion of glucose into dogs in which changes in plasma insulin and glucagon were prevented nonetheless resulted in a significant increase in VLDL-TG secretion (18).

The first step in determining the respective roles of chronic elevations in insulin and glucose in the kinetics of VLDL-TG in human subjects is to establish a model in which hyperinsulinemia is associated with hypertriglyceridemia and to determine the relative contributions of preformed and de novo synthesized FA to the accelerated rate of VLDL-TG production in that circumstance. To accomplish this goal, we have used a chronic (4-d) hypercaloric high-carbohydrate feeding regimen that produces hyperinsulinemia and hyperglycemia. We have used a new model of lipid kinetics based on the mass isotopomer distribution analysis technique (19-21) to quantify the major pathways of VLDL-TG production (secretion of preformed FA and secretion of de novo synthesized FA) and the

1. Abbreviations used in this paper: FRS, fractional rate of secretion; FSR, fractional synthesis rate; FTR, fractional turnover rate; $R_{\mathrm{a}}$, rate of appearance; TG, triglycerides. 
total rate of VLDL-TG secretion. We studied the subjects before and 1 and $4 \mathrm{~d}$ after the start of the hypercaloric carbohydrate diet in order to document possible changes which may occur over time in the effectiveness of endogenous insulin to stimulate glucose uptake and to suppress lipolysis.

\section{Methods}

Subjects. Five normal male volunteers (age $31 \pm 4$ ), three black and two white, participated in the study. They were healthy, as indicated by medical history and physical examination, were of normal weight (body mass index), were taking no medications, and had normal blood chemistries (liver function tests, thyroid function test, plasma glucose, plasma insulin or serum lipids) and hematologic parameters. The subjects were admitted on two occasions to the Clinical Research Center, University of Texas Medical Branch at Galveston, for the performance of the experiments. The study was approved by the institutional review board of University of Texas Medical Branch at Galveston. Informed consent was obtained for all procedures.

Experimental protocol. Three tracer infusion studies were performed on each subject; after an overnight fast (basal state) and after 1 and $4 \mathrm{~d}$ of hypercaloric carbohydrate feeding (days 1 and 4) (Fig. 1). The studies were performed no less than $3 \mathrm{~d}$ apart to allow for isotope washout. The basal state value was determined in the first session. The values after 1 (day 1) and $4 \mathrm{~d}$ (day 4 ) of hypercaloric carbohydrate feeding were determined in the second session.

The subjects were admitted to the Clinical Research Center the night before the first infusion study. They were given a standard meal and then no more meals throughout the study. The next day at 7 a.m. a constant infusion of glucose at $2 \mathrm{mg} / \mathrm{kg}$ per min was started through a catheter placed in the antecubital vein (Fig. 1). Glucose was infused to promote a measurable rate of hepatic FA synthesis, which was required by the model. This infusion rate was chosen because it is well below the maximal capacity for glucose oxidation in humans $(22,23)$ and allowed us to provide the subjects with $\sim 50 \%$ of their estimated

\section{General experimental design}

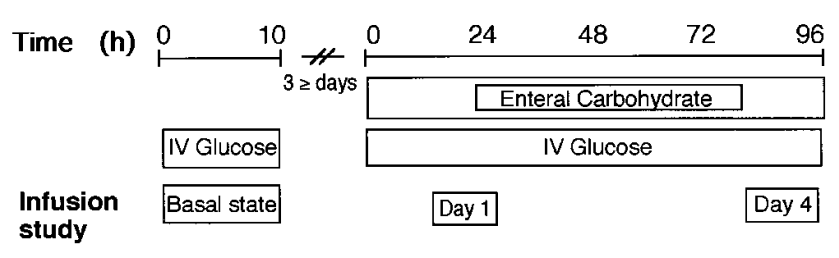

\section{Infusion protocol}

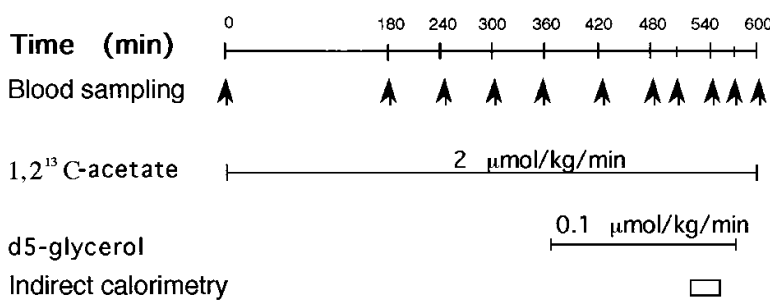

Figure 1. Experimental design. Each subject underwent a study in the basal state and during short (day 1) and prolonged (day 4) carbohydrate hyperalimentation. The studies were performed no less than $3 \mathrm{~d}$ apart to allow for isotope washout. In each study, labeled acetate was infused intravenously for $600 \mathrm{~min}$ to measure VLDL-TG kinetics and labeled glycerol was infused during the last $210 \mathrm{~min}$ to measure rate of adipose tissue lipolysis. Indirect calorimetry was used to calculate rate of fat oxidation. energy demands during the study. The isotope infusion was started at 8 a.m. (Fig. 1). After completion of this basal state study, the subjects were discharged.

For the second infusion study the subjects were admitted at noon the day before the infusion study. They had been instructed not to eat on the day of admission. At 2 p.m. an 8 french nasogastric feeding tube was placed into the upper gastrointestinal tract. Proper positioning was confirmed by auscultation and aspiration of gastric fluid. At 3 p.m. a constant infusion of intravenous glucose at $2 \mathrm{mg} / \mathrm{kg}$ per min was started through a catheter placed in the antecubital vein. At the same time, nasogastric feeding with a liquid meal (Vivonex; Sandoz Nutrition Corp., Minneapolis, MN) was started. Vivonex had been diluted to a caloric density of $1 \mathrm{kcal} / \mathrm{ml}(80.4 \mathrm{grams} / 250 \mathrm{ml}$ water; total volume $300 \mathrm{ml}$ ) with an energy distribution of $83 \%$ carbohydrates, $15 \%$ amino acids, and $2 \%$ fat (linoleic acid). The carbohydrates were derived from partially hydrolyzed corn starch. A comparable diet has been shown to eliminate the influx of chylomicrons into circulation during feeding (24). The feeding was started at an initial rate of $50 \mathrm{ml} / \mathrm{h}$ and gradually increased over the next $6 \mathrm{~h}$ to a final rate of $2 \mathrm{ml} / \mathrm{kg} / \mathrm{h}$, i.e., $50 \mathrm{kcal} \cdot \mathrm{kg}^{-1} \mathrm{~d}^{-1}$. The nasogastric feeding and intravenous glucose infusion was maintained night and day throughout the study (Fig. 1). Thus, the background ${ }^{13} \mathrm{C}$ enrichment was constant during the tracer infusion. At the final rate a total of $8.7 \mathrm{mg} / \mathrm{kg} / \mathrm{min}$ of carbohydrate was provided; $6.7 \mathrm{mg} / \mathrm{kg} / \mathrm{min}$ was delivered through the nasogastric tube and $2 \mathrm{mg} / \mathrm{kg} / \mathrm{min}$ through the intravenous infusion. The total amount of calories provided was $\approx 60 \mathrm{kcal} / \mathrm{kg} / \mathrm{d}$ with $\sim 90 \%$ from carbohydrates. In addition, the subjects had free access to carbohydrate containing liquids without caffeine. This intake was not recorded since it did not exceed $1-4 \%$ of the daily caloric intake.

The carbohydrate delivery rate, based upon our previous longterm glucose infusion studies (22), was chosen in order to elicit an insulin response sufficient to maintain the plasma insulin concentration between 50 and $70 \mu \mathrm{U} / \mathrm{ml}$.

Tracer infusion. Teflon catheters were placed percutaneously into an antecubital vein, and a sampling catheter was inserted in a dorsal hand vein of the contralateral arm. The heated hand technique was used to obtain arterialized blood samples (25). After a blood sample was drawn to determine background enrichment, a constant infusion of $\left[1,2-{ }^{13} \mathrm{C}\right]$ acetate $(99 \%$ enriched; Isotec Inc., Miamisburg, $\mathrm{OH} ; 2 \mu \mathrm{mol} / \mathrm{kg} \cdot \mathrm{min}$, no prime) was started at $8 \mathrm{a} . \mathrm{m} .6 \mathrm{~h}$ into the infusion study (2 p.m.) a primed constant infusion of $\left[{ }^{2} \mathrm{H}_{5}\right]$ glycerol $(98 \%$ enriched; Cambridge Isotope Laboratories, Andover, MA; $0.1 \mu \mathrm{mol} /$ $\mathrm{kg} \cdot \mathrm{min}$; prime $1.5 \mu \mathrm{mol} / \mathrm{kg}$ ) was started. The exact infusion rate for glycerol was determined by measuring the concentration in the infusate. Both isotopes were infused using calibrated syringe pumps (Harvard Apparatus, Natick, MA).

Blood sampling. Blood to measure VLDL-TG kinetics was taken $0,180,240,300,360,420,480,540$, and $600 \mathrm{~min}$ after the beginning of the infusion study and placed into $10-\mathrm{ml}$ vacutainers containing disodium EDTA. Blood to measure glycerol kinetics was taken 480, 510, 540 , and $600 \mathrm{~min}$ after the beginning of the infusion study $(120,150$, 180, and $210 \mathrm{~min}$ after start of labeled glycerol infusion) and placed into $10-\mathrm{ml}$ vacutainers containing lithium heparin. All samples were placed on ice. Plasma was separated by centrifugation shortly after sampling. Samples for VLDL isolation were stored at $4^{\circ} \mathrm{C}$ until they were processed the next morning. All other samples were stored at $-20^{\circ} \mathrm{C}$ until they were processed.

Sample analysis. VLDL was isolated from $3 \mathrm{ml}$ of plasma by overlaying the plasma with a density $=1.006$ solution $(0.9 \% \mathrm{NaCl})$ and spinning it in a 70.1 Ti rotor at 50,000 $\mathrm{rpm}(171,500 \mathrm{~g}$ average $)$ for $20 \mathrm{~h}$ at $15^{\circ} \mathrm{C}$ in an ultracentrifuge (model L7-55; Beckman Instruments, Palo Alto, CA). After ultracentrifugation, the VLDL-TG was carefully removed along with the density solution found on top of the tube by the slicing tube technique $(26,27)$. The total volume of the resulting VLDL suspension was $3 \mathrm{ml}$.

TG concentration in the VLDL suspension and in plasma was determined enzymatically (RA-500; Technicon Instruments Corp., Tarrytown, NY) (28). Plasma glucose and lactate concentrations were 
measured using an automated analyzer (Yellow Springs Instrument Inc., Yellow Springs, OH). Plasma insulin concentration was measured by radioimmunoassay (Instar Corp., Stillwater, MI).

TG in the VLDL suspension were isolated by thin-layer chromatography, hydrolyzed to FFA, and derivatized to FA methyl esters. Relative concentrations of individual FAs in the VLDL fraction were determined by gas chromatography (model 5890; Hewlett-Packard Co., Palo Alto, CA). Isotopic enrichment of selected FAs was determined by gas chromatography-mass spectrometry (GC-MS) (model 5992; Hewlett-Packard Co.) in the electron impact ionization mode for the ultimate computation of the tracer/tracee ratio. For the palmitate methyl ester the ions of mass-to-charge ratio $(\mathrm{m} / \mathrm{e}) 270,271,272$, 273 , and 274 were selectively monitored, for the methyl ester of oleate the ions of mass-to-charge ratio $(\mathrm{m} / \mathrm{e})$ 296, 297, 298, 299, and 300 were monitored, and for the methyl ester of stearate the ions of massto-charge ratio $(\mathrm{m} / \mathrm{e}) 298,299,300,301$, and 302 were monitored. Isotopic enrichment of glycerol was determined by GC-MS (model 5985; Hewlett-Packard Co.) by following previously described procedures (29). Ions of $m / e 205$ and 208 were monitored.

Calculations. Rate of appearance $\left(R_{\mathrm{a}}\right)$ of glycerol was calculated with use of a steady state equation since sample analysis indicated that a physiological and isotopic steady state existed during infusion period (30). Glycerol $R_{\mathrm{a}}$ was used as a reflection of lipolysis, with the assumption that all glycerol released in the process of lipolysis, whether in the adipose tissue or in muscle tissue, appears in the plasma. This is because glycerol reutilization requires phosphorylation via the enzyme glycerol kinase, and this occurs mainly in the liver (31). Also, glycerol cannot be produced in the body, other than from lipolysis (32), and the results of this study showed that the contribution of the hydrolysis of plasma TG to $R_{\mathrm{a}}$ glycerol could not account for $>10 \%$ of $R_{\mathrm{a}}$ glycerol, and in most cases much less than that. Consequently, the glycerol $R_{\mathrm{a}}$ equals total lipolysis, and FFA release has been assumed to be three to one for every glycerol released since there is essentially no partial hydrolysis of TG. Any release of glycerol by omental adipocytes that is cleared on the first pass of the liver would cause an underestimation of whole body lipolysis with this methodology. However, evidence from dogs indicates that the amount of glycerol released into the portal vein that is cleared on the first pass of the liver is a small percentage of total $R_{\text {a }}$ glycerol (33). Although there may be a difference between dogs and humans, our subjects were lean and had normal body mass index, so it is reasonable to presume that they did not have a disproportionately high omental compared with whole body lipolytic activity. With these assumptions in mind, the $R_{\text {a }}$ glycerol is used to calculate the lower limit for whole body lipolysis.

Measurement of the rate at which de novo synthesized palmitate is secreted as VLDL-TG. The enrichment (p) of the intrahepatic precursor pool for FA synthesis (hepatic acetyl-CoA) was deduced according to the mass isotopomer method as initially outlined by Hellerstein et al. $(19,34)$ with some later modifications $(35)$. To calculate the fractional rate of secretion of VLDL-bound palmitate (FRS), we used the formula FSR $=\left[\left(E_{(\mathrm{t} 2)}-E_{(\mathrm{t} 1)}\right) /\left(t_{2}-t_{1}\right)\right] /[8 p(1-$ $\left.p)^{7}\right]$; where $t_{1}$ and $t_{2}$ are the times when samples are taken and $\mathrm{E}_{(\mathrm{t})}$ is the doubly labeled enrichment at time t. FSR is thus defined as the fraction of the plasma VLDL-bound palmitate pool, per unit of time, that is newly synthesized.

The differences between this formula and the traditional formula (36) for calculation of FSR are the factor of eight in the denominator and the factor $(1-p)^{7}$. The factor of eight accounts for the fact that it requires eight acetate molecules to form one palmitate molecule. The factor of $(1-p)^{7}$ accounts for the probability that seven acetate molecules which are not labeled will be incorporated into a palmitate molecule (35).

To obtain absolute synthesis rates, the fractional synthetic rate was multiplied by the pool size of VLDL-bound palmitate. The pool size was estimated by multiplying the measured value of VLDL-TG concentration by the relative concentration of palmitate in the VLDL-TG fraction times the assumed plasma volume of $37 \mathrm{ml} / \mathrm{kg}$ (37).

Measurement of total rate of VLDL-bound palmitate secretion. The total rate of VLDL-bound palmitate secretion rate is calculated from the measured rate at which de novo synthesized palmitate is secreted into the VLDL palmitate pool. Briefly, we can obtain the percentage of VLDL-bound palmitate which is derived from de novo produced palmitate (\% d.n.VLDL palmitate) from the extent of dilution which occurs between the precursor and the product. The specific formula is $\%$ d.n.VLDL palmitate $=[$ plateau enrichment of $m+$ 2 labeled palmitate $((8 \times p)] /(1-p)^{7}$.

The total rate of VLDL-bound palmitate secretion is then calculated by dividing the rate at which de novo synthesized palmitate is secreted as VLDL by the percentage of VLDL-bound palmitate which is derived from de novo produced palmitate (\% d.n.VLDL palmitate). If a plateau was not been reached during the infusion time, the extrapolated value for the plateau enrichment was used to calculate the total secretion rate.

Measurement of total VLDL-bound FA kinetics. Quantitatively, palmitate $(\mathrm{C} 16: 0)$, oleate $(\mathrm{C} 18: 1)$, stearate $(\mathrm{C} 18: 0)$, linoleate $(\mathrm{C} 18: 2)$, and palmitoleate (C16:1) make up $>97 \%$ of the FAs of VLDLbound TGs. To measure the total VLDL-bound TG secretion rate, the secretion rates of VLDL-bound stearate, oleate, and linoleate were measured principally as described for VLDL-bound palmitate except with minor modifications. In our calculations, the production and secretion of palmitoleate was considered to be the same as palmitate, and thus the two VLDL pools were combined in our calculations. This approach is justified by the fact that the synthesis of palmitoleate (C16:1) proceeds through the initial synthesis of palmitate (C16) and that the final desaturation step is the only differentiating step in their synthesis. Furthermore, during the study the concentration of the two FAs relative to each other remained constant in the FFA pool and the VLDL-TG pool. This suggests that the relative flux of the two FAs was the same throughout the study.

To differentiate between the secretion rate of de novo synthesized, elongated, or reesterified C18 FAs certain assumptions were made. It is known that palmitate is the primary product of de novo FA synthesis in the liver and that the C18 FAs such as stearate (C18: $0)$ and oleate $(\mathrm{C} 18: 1)$ are the products of elongation and desaturation of palmitate $(38,39)$. As such, the VLDL-bound stearate and oleate can principally come from three sources: from de novo synthesized palmitate (C16:0) with all its acetate molecules coming from the hepatic acetate pool and getting one more acetate unit to become stearate $(\mathrm{C} 18: 0)$ or undergoing further desaturation to become oleate (C18:1); from palmitate that is initially taken up from the plasma and elongated by the addition of one acetate molecule from the hepatic pool to become stearate and oleate; and from uptake from plasma and subsequent reesterification into VLDL-TG. In this study the term "de novo synthesized" is preserved for the first alternative, where all the acetate units of the FA are derived from the hepatic pool. The second and third alternatives are collectively termed reesterification. As such, reesterification encompasses both the process by which palmitate is taken up from plasma and elongated into a C18 FA for subsequent secretion and the process by which stearate and oleate (C18 FA) themselves are taken up from plasma and reesterified into VLDL-TG. Labeling of stearate and oleate with one labeled acetate molecule can be the consequence of elongation of any palmitate, de novo synthesized or not. C18 FAs containing multiple labeled acetate molecules $(2,3 \ldots .8)$ can only be the elongated product of de novo synthesized palmitate. To obtain more than one labeled acetate molecule, the C18 FA must have had labeled palmitate as the immediate precursor (palmitate with a minimum of one labeled acetate unit must be de novo synthesized since elongation of shorter chain FAs is not a major pathway [38]). For this reason, we calculated the FSR for the C18 FAs using their doubly labeled isotopomers (two labeled acetate molecules) rather than singly labeled stearate and oleate using the formula $F S R=\left[\left(E_{(12)}-E_{(11)}\right) /\left(t_{2}-t_{1}\right)\right] /\left[36 p^{2}(1-p)^{7}\right]$; where $t_{1}$ and $t_{2}$ are the times when samples are taken and $E_{(t)}$ is the doubly labeled enrichment at time t.

The denominator $36 p^{2}(1-p)^{7}$ represents the probability that two labeled acetate molecules will be incorporated into stearate or oleate $(\mathrm{m}+4$ FAs), given that the enrichment of the acetyl-CoA pool is $\mathrm{p}$. 
We used the value of $\mathrm{p}$ obtained from VLDL palmitate, since palmitate, stearate, and oleate are all derived from the same acetyl CoA pool. For subject MG on day 1 the $p$ value was obtained from VLDL stearate and not from VLDL palmitate because the palmitate had no detectable enrichment at this time point. Because stearate and oleate are synthesized from nine rather than eight acetyl-CoA molecules, the calculation of total VLDL-bound stearate and oleate secretion is otherwise the same as for palmitate except that percent VLDL is calculated using the formula $\% V L D L=$ plateau enrichment $/(9 \times$ precursor enrichment $) /(1-p)^{8}$.

Linoleate is an essential FA, meaning that it is not synthesized. Thus, the only quantity to be computed regarding linoleate is the secretion rate of VLDL-bound linoleate. This is computed by multiplying the VLDL palmitate secretion rate by the ratio of percent linoleate abundance to percent palmitate abundance in VLDL as determined by gas chromatography.

The fractional turnover rate (FTR) of the VLDL-TG pool was calculated by dividing the rate of secretion of VLDL-TG with the pool size of the VLDL-bound TGs, assuming that the pool size was constant. With the same assumption, VLDL-TG clearance rate was calculated as rate of uptake (= secretion) divided by the concentration of VLDL-TG.

Statistical analysis. All results are expressed as mean and SEM, except for the individually tabulated results. Comparisons of groups were performed by means of ANOVA. When ANOVA demonstrated statistical significance, Fisher's least significant difference test was used to compare differences between groups. Statistical significance was accepted at $\leq 0.05$.

\section{Results}

All subjects completed the study. The intravenous infusion and the nasogastric feeding (combined enteral and parenteral feeding) were maintained uninterrupted during the $4 \mathrm{~d}$ of the hyperinsulinemia period except for an interruption of $\sim 15$ min every morning to change infusion lines.

The ratio between erythrocyte volume and plasma volume, i.e., hematocrit, was unchanged during the study. This was expected, since the amount of blood withdrawn during each infusion study was $<2 \%$ of estimated total blood volume and this amount of blood loss should not have affected erythrocyte volume. Thus, plasma volume during the same period was assumed to be unchanged.

\section{Body weight, glucose, insulin, and lactate concentration}

The average body weight tended to increase throughout the study, from $73 \pm 3 \mathrm{~kg}$ in the basal state to $74 \pm 4$ and $75 \pm 4 \mathrm{~kg}$ after 1 and $4 \mathrm{~d}$ of hyperalimentation, respectively. This increase was not statistically significant. A slight but statistically insignificant increase in plasma glucose concentration was observed when the hypercaloric carbohydrate intake was started, i.e.,

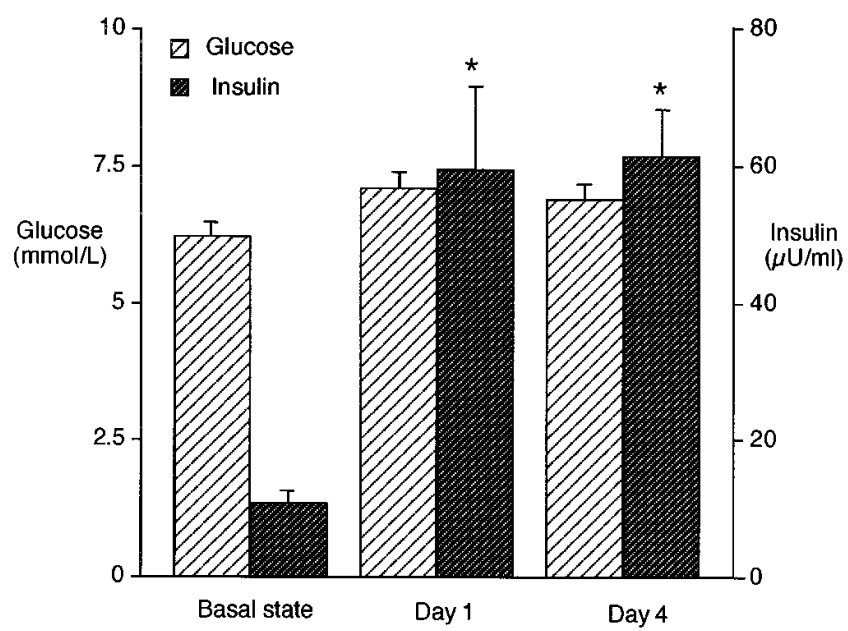

Figure 2. Glucose and insulin concentrations in plasma. *Significantly different at $P<0.05$ compared with the basal state.

going from basal state to day 1 (Fig. 2). During the ongoing hypercaloric carbohydrate intake, the plasma glucose concentration remained constant at a level of $\approx 7 \mathrm{mmol} / \mathrm{liter}$. The plasma insulin concentration was $10.8 \pm 1.5 \mathrm{mU} / \mathrm{ml}$ in the basal state. After $1 \mathrm{~d}$ of the combined enteral and parenteral hyperalimentation, the insulin concentration was elevated to $59.6 \pm 12 \mathrm{mU} / \mathrm{ml}$. It remained at this level of $\approx 60 \mu \mathrm{U} / \mathrm{ml}$ throughout the hypercaloric carbohydrate intake period (day 4) (Fig. 2). The plasma lactate concentration increased significantly during the study from an initial value of $1.0 \pm 0.06$ in the basal state to $1.6 \pm 0.18$ on day 1 and $2.6 \pm 0.46 \mathrm{mmol} / \mathrm{liter}$ on day 4 .

\section{Plasma lipids}

The average value for total plasma TG concentration was $0.92 \pm 0.2 \mathrm{mmol} \mathrm{TG} /$ liter during the basal state and increased slightly but not significantly during early carbohydrate hyperalimentation/hyperinsulinemia (day 1). After $4 \mathrm{~d}$ of carbohydrate hyperalimentation/hyperinsulinemia (day 4), total plasma TG concentration had increased significantly to $4.34 \pm 1.61$ mmol TG/liter (Table I).

The average VLDL-TG concentration increased slightly but not statistically from the basal state to day 1 (Table I). On day 1 , there was considerable individual variability. One subject had an $\approx 50 \%$ drop in VLDL-TG concentration (DM), two were unchanged (JW and MG), and two increased their VLDL-TG concentration $\approx 200 \%$ (DK and EB) (Data not

Table I. Concentrations, Pool Sizes, and Distribution of Plasma Lipids after 1 and 4 d of Hyperinsulinemia/Hyperglycemia $( \pm$ SEM)

\begin{tabular}{|c|c|c|c|}
\hline & Basal state & Day 1 & Day 4 \\
\hline Plasma TG (mmol/liter) & $0.92 \pm 0.22$ & $1.21 \pm 0.33$ & $4.34 \pm 1.61^{* \ddagger}$ \\
\hline VLDL TG (mmol/liter) & $0.30 \pm 0.08$ & $0.46 \pm 0.22$ & $2.93 \pm 1.22 * \sharp$ \\
\hline VLDL palmitate pool $(\mu \mathrm{mol} / \mathrm{FA} / \mathrm{kg})$ & $11.8 \pm 3.3$ & $24.2 \pm 13.1$ & $151 \pm 69.1^{* \ddagger}$ \\
\hline VLDL-TG pool $(\mu \mathrm{mol} \mathrm{TG} / \mathrm{kg})$ & $11.0 \pm 3.0$ & $17.2 \pm 8.2$ & $105.6 \pm 45.3^{* \neq}$ \\
\hline VLDL-TG \% of plasma TG & $31 \pm 5$ & $35 \pm 8 \%$ & $65 \pm 5 \% * \neq$ \\
\hline
\end{tabular}

$* P \leq 0.05$ statistically different from day $0 ;{ }^{\ddagger} P \leq 0.05$ different from day 1 . 


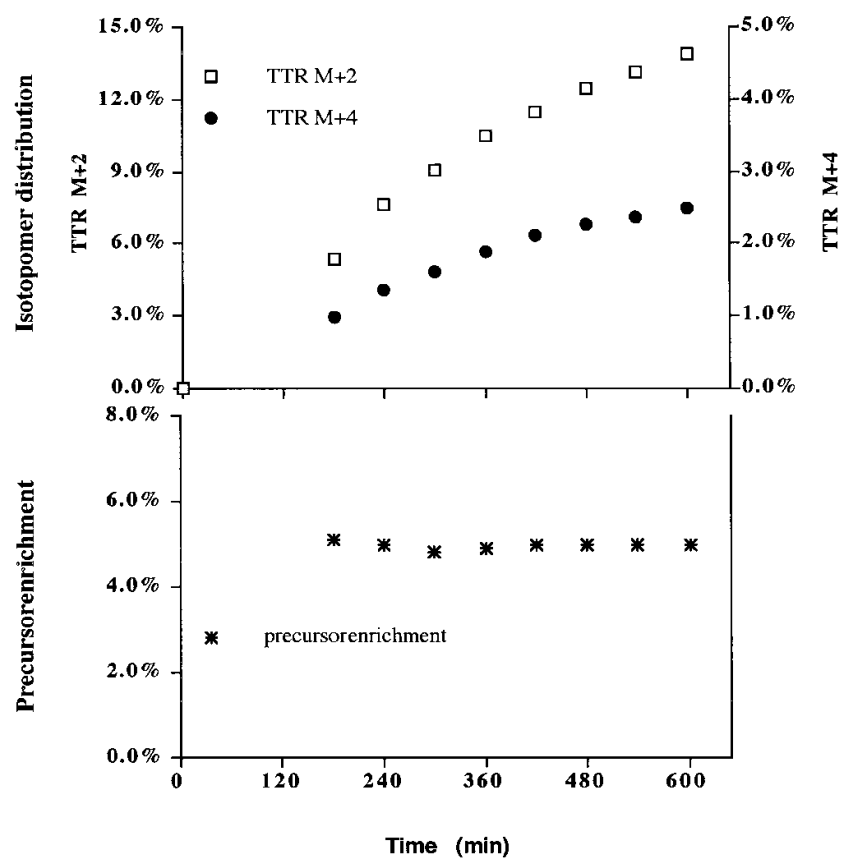

Figure 3. Isotopomer distribution and precursor enrichment. The top shows a representative example of the isotopomer distribution of VLDL palmitate during infusion of labeled acetate, i.e., the tracer/ tracee ratio $(T T R)$ for $M+2(m+2 / m+0)$ and $M+4(m+4 / m+$ $0)$ in an individual subject. The bottom shows the enrichment of the intrahepatic precursor pool for FA synthesis (acetyl-CoA) which is deduced based on the observed isotopomer distribution.

shown). After $4 \mathrm{~d}$ of carbohydrate hyperalimentation/hyperinsulinemia (day 4), all of the subjects had increased VLDL-TG concentration, and the average increased from $0.30 \pm 0.08$ $\mathrm{mmol} \mathrm{TG} / \mathrm{liter}$ on day 0 to $2.93 \pm 1.22 \mathrm{mmol} \mathrm{TG} / \mathrm{liter}$ on day 4 (Table I).

The size of the VLDL-TG pool size was calculated from the measured VLDL-TG concentration multiplied by assumed plasma volume $(37 \mathrm{ml} / \mathrm{kg}$ ) and normalized for body weight. Initially the VLDL-TG pool size was $11.0 \pm 3.3 \mu \mathrm{mol} \mathrm{TG} / \mathrm{kg}$. After $4 \mathrm{~d}$ of carbohydrate hyperalimentation/hyperinsulinemia (day 4), the pool size had increased $\sim 10$-fold to $105.6 \pm 45.3$ $\mu \mathrm{mol} \mathrm{TG} / \mathrm{kg}$. Before and after $24 \mathrm{~h}$ of carbohydrate hyperalimentation/hyperinsulinemia (day 1), VLDL-TGs accounted for $\sim 30 \%$ of total TGs. By the end of the prolonged hyperinsulinemic period (day 4), VLDL-TGs made up $65 \%$ of total plasma TGs.

\section{VLDL-bound palmitate kinetics}

The doubly labeled and quadruply labeled mass isotopomers, i.e., the $\operatorname{TTR}(\mathrm{M}+2)$ and $\operatorname{TTR}(\mathrm{M}+4)$ ratios, were used to calculate the kinetics of VLDL-bound palmitate. Fig. 3 shows a representative example of the distribution of the two mass isotopomers of palmitate during the 10 -h infusion of labeled acetate. If either one of the enrichment curves are extrapolated to $0 \%$ enrichment, they do not intercept at time point 0 but at some later time. This time represents the delay between the start of the labeled acetate infusion and the first appearance of enriched de novo synthesized palmitate in the VLDL pool. An average delay time of $\sim 30$ min was found during all three infusion studies (data not shown).

The precursor enrichment for de novo synthesized palmitate (intrahepatic acetate pool) is shown in Fig. 3. An infusion rate of $2 \mu \mathrm{mol} / \mathrm{kg} / \mathrm{min}$ of $\left[1,2-{ }^{13} \mathrm{C}\right]$ acetate gave an average precursor enrichment in the basal state of $8 \pm 1.8 \%, 7 \pm 0.8 \%$ at day 1 and $5 \pm 0.5 \%$ at day 4 .

The fractional rate of secretion (FRS) of de novo synthesized palmitate bound to VLDL was calculated based on the precursor enrichment and the slope of the linear segment of the $\mathrm{M}+2$ isotopomer enrichment curve $[\operatorname{TTR}(\mathrm{M}+2)$ vs. time] (Fig. 3). The FRS was $23 \pm 10 \% / d$ of the VLDL-bound palmitate pool in the basal state. After $1 \mathrm{~d}$ of carbohydrate hyperalimentation, it increased 5.6-fold to $129 \pm 40 \% /$ d (day 1) and after prolonged hyperalimentation remained significantly increased at a level 3.4-fold higher than the basal state value (day 4) (Table II). To obtain the absolute rate of secretion, the FRS of de novo synthesized palmitate was multiplied by the pool size of VLDL-bound palmitate (Table I). The absolute rate of secretion of de novo synthesized palmitate increased significantly throughout the hypercaloric carbohydrate intake from a basal state value of $2.6 \pm 1.2$ to $40.8 \pm 20.0$ at day 1 and finally to $133.3 \pm 42.0 \mu \mathrm{mol} F A / \mathrm{kg} / \mathrm{d}$ at day 4 . The total rate of secretion of palmitate bound to VLDL (from both de novo synthesized and preformed FA) was not significantly affected by the early carbohydrate feeding but increased significantly after $4 \mathrm{~d}$ of feeding from a value of $58 \pm 24 \mu \mathrm{mol} \mathrm{FA} / \mathrm{kg} / \mathrm{d}$ at the basal state to $346 \pm 121 \mu \mathrm{mol} \mathrm{FA} / \mathrm{kg} / \mathrm{d}$ at day 4 .

\section{Secretion of VLDL-TG}

Rate of secretion of total VLDL-TG. In the basal state the rate of secretion of VLDL-TG was $72 \pm 23 \mu \mathrm{mol} \mathrm{TG} / \mathrm{kg} / \mathrm{d}$ (Table III). After $1 \mathrm{~d}$ of carbohydrate hyperalimentation/hyperinsulinemia (day 1), the total secretion rate was decreased to $52 \pm 24 \mu \mathrm{mol} \mathrm{TG} / \mathrm{kg} / \mathrm{d}$ but this did not reach statistical significance $(P \leq 0.05)$. As in the case of VLDL-TG concentration, there was a marked individual difference in response to carbo-

Table II. Parameters Used to Calculate VLDL-bound Palmitate Kinetics after 1 and 4 d of Hyperinsulinemia/Hyperglycemia $( \pm$ SEM)

\begin{tabular}{|c|c|c|c|}
\hline & Basal state & Day 1 & Day 4 \\
\hline Precursor enrichment $(m+2 / m+0$ acetate $\times 100)$ & $8.1 \pm 1.8 \%$ & $7.2 \pm 0.7 \%$ & $4.8 \pm 0.5 \% *$ \\
\hline Plateau enrichment $t^{\ddagger}(m+2 / m+0$ palmitate $\times 100)$ & $2.1 \pm 1.4 \%$ & $18.7 \pm 2.0 \% \S$ & $8.1 \pm 0.3 \% * \S$ \\
\hline Fractional secretion rate of de novo synthesized palmitate (\%/d) & $23 \pm 10 \%$ & $129 \pm 40 \%{ }^{\S}$ & $79 \pm 9 \%{ }^{\S}$ \\
\hline Absolute secretion rate of de novo synthesized palmitate ( $\mu \mathrm{mol}$ palmitate $/ \mathrm{kg} / \mathrm{d}$ ) & $2.6 \pm 1.2$ & $40.8 \pm 20.0^{\S}$ & $133.3 \pm 42.0 * 8$ \\
\hline Total rate of secretion $(\mu \mathrm{mol}$ palmitate $/ \mathrm{kg} / \mathrm{d})$ & $58 \pm 24$ & $75 \pm 38$ & $346 \pm 121 * \S$ \\
\hline
\end{tabular}

${ }^{\S} P \leq 0.05$ statistically different from day $0 ; * P \leq 0.05$ different from day $1 .{ }^{*}$ Plateau enrichment $=$ TTR $(\mathrm{M}+2)$ enrichment of VLDL-bound palmitate at plateau either as observed or extrapolated if no plateau had been reached. 
Table III. Total Hepatic Secretion of VLDL-TG after 1 and $4 d$ of Hyperinsulinemia/Hyperglycemia ( $\mu \mathrm{mol} \mathrm{TG} / \mathrm{kg} / \mathrm{d}$ )

\begin{tabular}{lccc}
\hline \multicolumn{1}{c}{ Subjects } & Basal state & Day 1 & Day 4 \\
\hline DM & 160 & 6 & 160 \\
DK & 63 & 39 & 547 \\
MG & 67 & 12 & 88 \\
JW & 24 & 33 & 176 \\
EB & 45 & 72 & 240 \\
Average $( \pm$ SEM $)$ & $72 \pm 23$ & $52 \pm 24$ & $242 \pm 78^{*}$
\end{tabular}

${ }^{*} P \leq 0.05$ statistically different from day $0 ;{ }^{\ddagger} P \leq 0.05$ different from day 1 .

hydrate hyperalimentation/hyperinsulinemia at this time point. After $4 \mathrm{~d}$ of carbohydrate hyperalimentation/hyperinsulinemia, the VLDL-TG secretion rate was elevated in all subjects. The average rate was $242 \pm 78 \mu \mathrm{mol} \mathrm{TG} / \mathrm{kg} / \mathrm{d}$, which is a 3.4fold increase as compared with rates in the basal state.

Rate of secretion of VLDL-TG derived from preformed $F A$. The initial response (day 1 ) to carbohydrate hyperalimentation/hyperinsulinemia was a decrease in the rate of secretion of VLDL-TG that was derived from preformed FA. However, after $4 \mathrm{~d}$ of carbohydrate hyperalimentation/hyperinsulinemia this response was reversed to a 2.7 -fold increase in the rate of secretion of preformed FA (Table IV).

Rate of secretion of VLDL-TG derived from de novo synthesized FA. In Table V, the rates of secretion of VLDL-TG derived from de novo synthesized FA are presented for each subject as observed before (basal state) and during carbohydrate hyperalimentation/hyperinsulinemia (days 1 and 4). After $1 \mathrm{~d}$ of carbohydrate hyperalimentation/hyperinsulinemia, the average secretion of de novo synthesized fat was significantly increased from $1.1 \pm 0.4$ in the basal state to $15.9 \pm 7.9$ $\mu \mathrm{mol} \mathrm{TG} / \mathrm{kg} / \mathrm{d}$ (day 1 ). On the fourth day of carbohydrate hyperalimentation/hyperinsulinemia (day 4), the average secretion rate of de novo synthesized FA was $50.0 \pm 18.8 \mu \mathrm{mol} \mathrm{TG} /$ $\mathrm{kg} / \mathrm{d}$ (Table V).

Turnover time and clearance of VLDL-TG, peripheral lipolysis, and lipid oxidation

In the basal state the FTR of the VLDL-TG pool was 9.2 \pm 3.8 pools/d. Hyperinsulinemia slowed down the FTR of the VLDL-TG pool to $2.1 \pm 0.2$ pools/d. $4 \mathrm{~d}$ of carbohydrate hyperalimentation/hyperinsulinemia (day 4) further reduced the

Table IV. Hepatic Secretion of VLDL-TG Derived from Preformed FA after 1 and 4 d of Hyperinsulinemial Hyperglycemia ( $\mathrm{mol} \mathrm{TG} / \mathrm{kg} / \mathrm{d}$ )

\begin{tabular}{lccc}
\hline \multicolumn{1}{c}{ Subjects } & Basal state & Day 1 & Day 4 \\
\hline DM & 159 & 5 & 136 \\
DK & 62 & 96 & 415 \\
MG & 66 & 9 & 76 \\
JW & 21 & 24 & 135 \\
EB & 44 & 49 & 191 \\
Average $( \pm$ SEM) & $70 \pm 23$ & $37 \pm 16$ & $191 \pm 57^{* \neq}$ \\
& & & \\
\hline
\end{tabular}

$* P \leq 0.05$ statistically different from day $0 ;{ }^{*} P \leq 0.05$ different from day 1 .
Table V. Hepatic Secretion of VLDL-TG Derived from De Novo Synthesized FA after 1 and $4 d$ of Hyperinsulinemial Hyperglycemia ( $\mu \mathrm{mol} \mathrm{TG} / \mathrm{kg} / \mathrm{d})$

\begin{tabular}{lccr}
\hline \multicolumn{1}{c}{ Subjects } & Basal state & Day 1 & Day 4 \\
\hline DM & 1.3 & 1.0 & 27.6 \\
DK & 0.3 & 43.5 & 120.7 \\
MG & 1.0 & 2.9 & 11.9 \\
JW & 2.4 & 9.1 & 41.1 \\
EB & 0.4 & 22.9 & 48.9 \\
Average (土SEM) & $1.1 \pm 0.4$ & $15.9 \pm 7.9^{*}$ & $50.0 \pm 18.8^{* \ddagger}$ \\
& & & \\
\hline
\end{tabular}

$* P \leq 0.05$ statistically different from day $0 ;{ }^{\ddagger} P \leq 0.05$ different from day 1 .

FTR to $2.1 \pm 0.3$ pools/d (Table VI). The VLDL-TG clearance rate was also lowered by both acute (day 1) as well as prolonged (day 4) carbohydrate hyperalimentation/hyperinsulinemia. The clearance rate was significantly lowered from $0.35 \pm 0.08 \mathrm{liter} / \mathrm{kg} / \mathrm{d}$ in the basal state to $0.11 \pm 0.02$ and $0.09 \pm 0.01 \mathrm{liter} / \mathrm{kg} / \mathrm{d}$ at days 1 and 4 , respectively. There was a slight decrease in the rate of appearance of glycerol $\left(R_{\mathrm{a}}\right.$ glycerol) initially (day 1 ) and a return to normal by day 4 .

\section{Discussion}

Hypertriglyceridemia is commonly associated with insulinresistant states, such as type II diabetes (1-3). However, the underlying mechanisms responsible for the apparent relationship between increases in insulin and TGs are unclear, in part because previously it has not been possible to create an experimental model in normal volunteers. In this experiment, we established such a model by the prolonged (4-d) continuous ingestion and infusion of a hypercaloric carbohydrate diet that produced chronic elevations in both plasma insulin and glucose concentration. In this circumstance, the plasma TG concentration was elevated fourfold as compared with the values in the basal state, due to both an increase in VLDL-TG secretion and a decrease in VLDL-TG clearance. The accelerated VLDL-TG production resulted primarily from increased secretion of preformed FA, and to a lesser extent from increased secretion of de novo synthesized FA. The length of time of dietary treatment was important. Thus, in contrast to the response on day 4, after $1 \mathrm{~d}$ of diet VLDL-TG production was lower than the basal state. Changes in the channeling of preformed FA towards VLDL-TG production were prominent in explaining the difference between the response on days 1 and 4. After $1 \mathrm{~d}$ of hypercaloric carbohydrate intake, VLDL-TG output coming from preformed FA was reduced, and on day 4 the secretion of preformed FA was significantly accelerated.

Tracer technique. We have used a new approach to quantify VLDL-TG kinetics. The starting point is the calculation of the rate of de novo FA synthesis according to the mass isotopomer distribution analysis, fundamentally as described by Hellerstein et al. $(19,34)$. Recently, we have discussed the details of the calculations and assumptions involved in this calculation (35). The conceptual breakthrough of this methodology is that the true precursor enrichment is determined by analysis of the product, thereby eliminating any assumptions regarding the unknown extent of intracellular dilution of the precursor enrichment, as measured in plasma. Furthermore, by measur- 
Table VI. Lipid Kinetics after 1 and 4 d of Hyperinsulinemial Hyperglycemia $( \pm S E M)$

\begin{tabular}{|c|c|c|c|}
\hline & Basal state & Day 1 & Day 4 \\
\hline $\begin{array}{l}\text { Fractional turnover rate of } \\
\text { VLDL-TG (pools/d) }\end{array}$ & $9.2 \pm 3.8$ & $2.1 \pm 0.2^{*}$ & $2.1 \pm 0.3^{*}$ \\
\hline $\begin{array}{l}\text { Rate of clearance of VLDL-TG } \\
\text { (liters } / \mathrm{kg} / \mathrm{d})\end{array}$ & $0.35 \pm 0.08$ & $0.11 \pm 0.02 *$ & $0.09 \pm 0.01 *$ \\
\hline $\begin{array}{l}\text { Total peripheral lipolysis }{ }^{\ddagger} \\
(\mathrm{mmol} \mathrm{TG} / \mathrm{kg} / \mathrm{d})\end{array}$ & $3.37 \pm 0.68$ & $2.55 \pm 0.5$ & $4.13 \pm 1.57$ \\
\hline $\begin{array}{l}\text { Net whole body TG oxidation } \\
(\mathrm{mmol} \mathrm{TG} / \mathrm{kg} / \mathrm{d})\end{array}$ & $1.10 \pm 0.34$ & $0^{*}$ & $0 *$ \\
\hline
\end{tabular}

¥Total peripheral lipolysis is based on $R_{\mathrm{a}}$ glycerol where one glycerol molecule is assumed to represent the hydrolysis of one TG molecule. ${ }^{*} P \leq 0.05$ statistically different from day $0 ;{ }^{\S} P \leq 0.05$ different from day 1 .

ing the isotopic enrichment in multiple FAs, it was unnecessary to make assumptions regarding the extrapolation of the kinetics of one FA (e.g., palmitate) to all the FAs. The kinetics of the VLDL-TG was computed by combining the FSR of the individual VLDL FAs with their respective VLDL pool sizes.

Several assumptions were necessary for our model. For the calculation of the total rate of VLDL-TG secretion, we assumed that VLDL-TG were distributed entirely in the plasma pool and that, over the course of each tracer infusion, the pool size was constant and reasonably estimated from literature values. Because of the size of the VLDL particle, it is reasonable to assume restriction to the plasma (26). The constant hematocrit indicated that the plasma volume was constant during the studies. Consequently, it is reasonable to assume that the plasma concentration of VLDL-TG was a direct reflection of the pool size. The concentration of VLDL normally fluctuates over the course of the day (26). Furthermore, since the concentration of VLDL progressively increased over days (Table I), in a general sense we can assume the pool size must have been increasing to at least some extent on day 1 . However, the time course of this increase was presumably slow, since over the time frame of our sampling, the concentration of VLDLTG was relatively constant.

The calculation of total VLDL-TG pool size required the assumption of a value for plasma volume. This was unlikely to introduce much error since the previously published range for plasma volume in normal man is $35-40 \mathrm{ml} / \mathrm{kg}$ and the coefficient of variation is $10 \%$ (37). This means that the error in the estimated volume of distribution was no more than $10 \%$, which would approximately correspond to maximum of a $10 \%$ error in the calculated rate of total VLDL-TG secretion. Importantly, there would not be a bias in the distribution of any errors stemming from this assumption.

It was also necessary to assume that there was no recycling of label back into TG. This is a reasonable assumption, since most FAs in the VLDL-TG come from plasma FFA and VLDL-TG contributes minimally to plasma FFA flux (18). The plasma palmitate $M+2$ enrichment was never $>10 \%$ of the enrichment measured for VLDL-bound palmitate and most frequently was found to be below detectable levels (data not shown). Furthermore, the isotopic equilibrium in the FA enrichment in the TG pool confirmed that there was minimal recycling of label, since the enrichment would have progres- sively increased if there had been a significant amount of recycling.

Label could potentially also recycle within the acetyl-CoA pool of the liver. By recycling of acetyl-CoA in the tricarboxylic acid cycle $M+1$ acetate could theoretically be generated from $M+2$ acetate. Subsequent incorporation of two $M+1$ acetate molecules into newly synthesized FA could lead to an overestimation of the $\mathrm{M}+2$ isotopomer thought to be the product of $\mathrm{M}+2$ acetate incorporation. The most likely event would be the incorporation of only one $\mathrm{M}+1$ acetate that would generate the $\mathrm{M}+1$ isotopomer of the FA. Apparently this did not take place to any significant extent. The $M+1$ enrichment of palmitate bound to VLDL-TG was never $>5 \%$ above the expected enrichment according to natural abundance of ${ }^{13} \mathrm{C}$. Thus, intrahepatic generation of $\mathrm{M}+1$ acetate in the tricarboxylic acid cycle with subsequent incorporation into newly synthesized FA appeared to be insignificant.

One advantage of the tracer model we used was that the rate of secretion of newly synthesized FA was determined without assumptions regarding precursor enrichment. On the other hand, the method was limited by the requirement for a certain amount of de novo synthesis of FA and the subsequent secretion of these FAs as VLDL-bound FA. For this reason, the initial basal study was performed during the infusion of glucose at $2 \mathrm{mg} / \mathrm{kg}$ per min, rather than in the fasting state, so that there would be at least a measurable rate of FA synthesis.

The total secretion rate of VLDL-bound FA was calculated based on the fractional secretion rate of newly synthesized FA that appear in VLDL-TG. The total rate of secretion of VLDL-TG was then calculated, based on the dilution principle from knowledge of the precursor enrichment and the product enrichment at isotopic plateau, and from estimation of the total VLDL-TG pool size. The rate of secretion of preformed FA was calculated as the difference between the total secretion rate and the rate of secretion of newly synthesized FA. The term preformed FA refers to the FAs that were not synthesized within the liver, but rather were derived from some other source of FA. Potential sources include the plasma FFA, lipoprotein remnants in plasma, or TG stored in the liver (intrahepatic lipolysis). We did not attempt to differentiate between potential intrahepatic pools of preformed FAs.

The plasma FFA pool is known to be the quantitatively most important contributor to the flux through the pathway of preformed FA in man (13). Although the role of a potential intrahepatic storage pool of TG is largely unknown in man, it may also play a role. In cultured hepatocytes it has been shown that intracellular TG formed directly from extracellular FA is stored temporarily within the cell and that FA derived from this TG store serve as the major substrate for VLDL-TG synthesis (40). The FA of the intrahepatic TG store can theoretically come from the diet (exogenous FA), from lipolysis (endogenous FA), or from FA synthesized in the liver (de novo FA). During our experiment, peripheral lipolysis was the major source of plasma FFA and exogenous FA were not entering the system. The extent of entry of de novo synthesized FA into a TG storage pool during the $10 \mathrm{~h}$ of our experiment is unknown. However, the fact that an isotopic equilibrium in the FA enrichment in VLDL-TG was achieved indicates that if there was entry of newly produced, labeled FA into the intrahepatic TG pool, the label was leaving the pool at the same rate. Furthermore, $10 \mathrm{~h}$ has been shown previously to be a sufficient time for equilibration between the plasma FFA pool 
and the intrahepatic TG pool (15). Consequently, whereas some of the specific FA that were incorporated into the VLDL-TG in the course of the study may have come from the intrahepatic TG store, there was probably little net change in the intrahepatic TG pool size.

The validity of our method for quantifying VLDL-TG kinetics is supported by the agreement between our results and the results reported by others in comparable situations. VLDL-TG kinetics have been measured previously using tracers (41-43) as well as catheterization techniques $(13,44)$. Previously published rates for total VLDL-TG secretion rates in human subjects range from $100(24,45)$ to $400 \mu \mathrm{mol} \mathrm{TG} / \mathrm{kg} / \mathrm{d}$ $(43,44,46)$. Our observed rate of total VLDL-TG secretion of $52-242 \mu \mathrm{mol} \mathrm{TG} / \mathrm{kg} / \mathrm{d}$ (Table III) is thus within the range observed for normotriglyceridemic subjects. The subjects in our study all had initial TG concentrations in the lower 50th percentile (Table I), which may indicate that the selected subjects also had a secretion rate in the lower normal range (45). The FTR of the VLDL-TG pool in the basal state was also within the range of previously published results. In the basal state we found that the FTR of the VLDL-TG pool was $9 \mathrm{~d}^{-1}$ as compared with previously reported values of $3-14 \mathrm{~d}^{-1}(42,43$, $45,46)$.

Experimental model of hyperinsulinemia. Whereas we were successful in creating an experimental model in normal volunteers in which hyperinsulinemia was associated with hypertriglyceridemia, we cannot differentiate between the effects of hyperinsulinemia and hypercaloric carbohydrate intake. This is because of the inevitable link between an elevation in insulin concentration and the requirement for extra glucose to avoid hypoglycemia. This problem is not avoided with the traditional euglycemic-hyperinsulinemic clamp technique (47). In that case, extra glucose is required to maintain euglycemia, and thus even though plasma glucose concentration is maintained constant, the rate of glucose uptake increases markedly. Rather, we chose to give glucose continuously, both orally and intravenously, and thus the increase in insulin originated endogenously. This approach had the practical advantage that hyperinsulinemia could be maintained easily for several days. Furthermore, the entry of the glucose and insulin into the body via the portal circulation was physiological, thereby maximizing the extent of hepatic glucose uptake (48) and maintaining the normal portal vein/arterial gradient in insulin concentration. Both of these factors may be important in producing hypertriglyceridemia (1). Furthermore, hyperinsulinemia usually occurs clinically in states such as diabetes mellitus, in conjunction with an increase in glucose concentration (49). The moderately elevated TG concentrations that developed after $4 \mathrm{~d}$ of hyperinsulinemia in our study were within the range frequently found in subjects with glucose intolerance or early diabetes with maintained pancreatic secretory function (2). Similar to the hypertriglyceridemia of the insulin-resistant states, the predominant increase in TGs was in the VLDL fraction (Table I) (2). Collectively, these factors imply that our model encompasses essential aspects of the hyperinsulinemia that are associated with insulin-resistant states and the associated effects on lipid metabolism.

$V L D L-T G$ breakdown. The magnitude of reduction in VLDL clearance with carbohydrate hyperalimentation/hyperinsulinemia was about the same as the increase in secretion. Thus, impaired hydrolysis either as a consequence of an altered nature of the particles or a change in the removal mecha- nism of VLDL-TG played an important role in causing hypertriglyceridemia. Most likely the VLDL particle increased in size and TG content as hepatic TG secretion was increased (26) and this could cause a decrease in their rate of removal (50).

$V L D L-T G$ secretion during hyperinsulinemia. After $24 \mathrm{~h}$ of carbohydrate hyperalimentation/hyperinsulinemia, total secretion of VLDL-TG tended to decrease, but statistically this trend was not significant due to divergent individual responses. This variability in response is understandable when the individual pathways are considered, because the total secretion rate after $24 \mathrm{~h}$ of carbohydrate hyperalimentation/hyperinsulinemia was a function of two opposing trends (Tables IV and V). The decreased secretion of preformed FA, coupled with an increase in de novo synthesis, could explain the conflicting results obtained in previous studies in which acute hyperinsulinemia either decreased $(12,15,50-53)$ or did not affect $(11,54)$ VLDL secretion. Depending on the experimental factors, such as the selection of subjects, whether or not they were fasted, and in particular the duration and extent of hyperinsulinemia, the effect on one pathway may predominate.

The results obtained on day 1 versus day 4 of carbohydrate hyperalimentation/hyperinsulinemia emphasize the importance of the duration of the hyperinsulinemia and probably reflect different time-dependent effects of insulin. On day 4, total VLDL-TG secretion rate was increased 3.4-fold as compared with the basal state. Whereas de novo synthesis was increased after $4 \mathrm{~d}$, the increase in VLDL-TG secretion was primarily due to stimulation of the secretion of preformed FA. The contrary was the case after short-term carbohydrate hyperalimentation. Total secretion of VLDL-TG tended to decrease on day 1 , probably because of an acute insulin-mediated inhibition of lipolysis that subsided as the hyperalimentation/ hyperinsulinemia persisted for an additional $3 \mathrm{~d}$ (Table VI).

A difference between acute and longer term effects of insulin on VLDL-TG secretion in isolated hepatocytes has been recognized $(10,54)$, but the relevance of those findings to the in vivo situation has been uncertain, because of the maintenance of a constant extracellular concentration of FFA in the in vitro circumstance. In human subjects, chronic hyperinsulinemia of endogenous origin, such as that observed in obesity, steroid use, or impaired glucose tolerance (55), has also been shown to be associated with increased VLDL-TG production. An excessive secretion of VLDL-TG has also been observed in the majority of studies of non-insulin-dependent diabetes mellitus (2). However, although hypertriglyceridemia is associated with increased postprandial insulin concentration (2) in non-insulin-dependent diabetes mellitus, insulin treatment has been shown to lower VLDL-TG secretion (50), raising the possibility that increased VLDL-TG secretion is not caused by hyperinsulinemia, per se, but rather is a phenomenon that accompanies insulin resistance.

It is well documented that under most circumstances plasma FFA serve as the predominant source of FAs for VLDL-TG. Even in the extreme circumstance of $4 \mathrm{~d}$ of excess carbohydrate intake we used in this study, which was designed to maximize the rate of de novo FA synthesis, the preformed FA were nonetheless the major source of VLDL-TG FAs. Thus, it is not surprising that the notion has been put forth that the rate of delivery of FAs to the liver, determined by the rate of peripheral lipolysis, is the predominant determinant of the rate of VLDL-TG secretion $(13,56)$. Whereas this may be true 
in some circumstances, this explanation cannot explain the hypertriglyceridemia after $4 \mathrm{~d}$ of hypercaloric carbohydrate intake in our experiment, because there was no significant increase in lipolysis (i.e., $R_{\text {a }}$ glycerol) going from the basal state to day 4. Rather, the partitioning of plasma FFA into TG and other pathways ( $\beta$-oxidation) in the liver is a more likely explanation of the time-dependent change in VLDL-TG output between days 0 and 4 that we observed (57). After $4 \mathrm{~d}$ of hypercaloric carbohydrate feeding, most likely the liver was deriving virtually all of its energy from carbohydrate oxidation as opposed to FA oxidation (58), meaning FA taken up by the liver would be channeled into VLDL-TG.

Based on these observations, we conclude that in our study during prolonged hyperinsulinemia the availability of preformed FA for TG synthesis was the major determinant of hepatic VLDL-TG secretion even though the availability of de novo synthesized FAs was increased severalfold by the prolonged intake of hypercaloric amounts of carbohydrates. Acute but not prolonged hyperinsulinemia associated with carbohydrate hyperalimentation has an inhibitory effect on the secretion of preformed FA reesterified into TG in the liver. Increased secretion and decreased catabolism of VLDL-TG was equally important in explaining the hypertriglyceridemia associated with the prolonged carbohydrate hyperalimentation/ hyperinsulinemia.

\section{Acknowledgments}

The authors would like to thank Dr. R.M. Carroll for methodological advice and the nurses of the Clinical Research Center, University of Texas Medical Branch for their assistance. We are indebted to Yunxia Lin for her technical assistance.

This study was supported by National Institutes of Health grants DK-34817 and DK-33952 and M01 RR00073, grant 15849 from the Shriners Hospital, and grants from The Norwegian Research Council.

\section{References}

1. Steiner, G. 1993. Hyperinsulinemia and VLDL kinetics. Adv. Exp. Med. Biol. 334:287-294.

2. Howard, B.V. 1987. Lipoprotein metabolism in diabetes mellitus. J. Lipid Res. 28:613-628.

3. Steiner, G. 1981. Diabetes and atherosclerosis: an overview. Diabetes. 30(Suppl. 2):1-7.

4. Taskinen, M.-R. 1993. Hyperinsulinism and dyslipidemias as coronary heart disease risk factors in NIDDM. Adv. Exp. Med. Biol. 334:295-300.

5. Carlson, L.A., L.E. Bottiger, and P.E. Ahfeldt. 1979. Risk factors for myocardial infarction in the Stockholm Prospective Study: a 14 year follow-up focusing on the role of plasma triglycerides and cholesterol. Acta. Med. Scand. 206:351-360.

6. Castelli, W.P. 1986. The triglyceride issue: a view from Framingham. Am. Heart J. 112:432-437.

7. Reaven, G.M., and Y.D.I. Chen. 1988. Role of insulin in regulation of lipoprotein metabolism in diabetes. Diabetes Metab. Rev. 4:639-652.

8. Taskinen, M.-R. 1987. Lipoprotein lipase in diabetes. Diabetes Metab. Rev. 3:551-570.

9. Berthezene, F. 1992. Hypertriglyceridemia: cause or consequence of insulin resistance? Horm. Res. 38:39-40.

10. Gibbons, G.F. 1989. Insulin, diabetes and hepatic very-low-density lipoprotein metabolism. Biochem. Soc. Trans. 17:49-51.

11. Woodside, W.F., and M. Heimberg. 1976. Effects of anti-insulin serum, insulin, and glucose on output of triglycerides and ketogenesis by the perfused rat liver. J. Biol. Chem. 251:13-23.

12. Shumak, S.L., B. Zinman, S. Zuniga-Guarjardo, M. Poapst, and G. Steiner. 1988. Triglyceride-rich lipoprotein metabolism during acute hyperinsulinemia in hypertriglyceridemic humans. Metab. Clin. Exp. 37:461-466.

13. Havel, R.J., J.P. Kane, E.O. Balasse, N. Segel, and L.V. Basso. 1970. Splanchnic metabolism of free fatty acids and production of triglycerides of very low density lipoproteins in normotriglyceridemic and hypertriglyceridemic humans. J. Clin. Invest. 49:2017-2035.
14. Coppack, S.W., M.D. Jensen, and J.M. Miles. 1994. In vivo regulation of lipolysis in humans. J. Lipid Res. 35:177-193.

15. Lewis, G.F, K.D. Uffelman, L. Szeto, B. Weller, and G. Steiner. 1995. Interaction between free fatty acids and insulin in the acute control of very low density lipoprotein production in humans. J. Clin. Invest. 95:158-166.

16. Kazumi, T., M. Vranic, and G. Steiner. 1986. Triglyceride kinetics: effects of dietary glucose, sucrose, and fructose alone or with hyperinsulinemia. Am. J. Physiol. 250:E325-E330.

17. Hellerstein, M.K., M. Christiansen, S. Kaempfer, C. Kletke, K. Wu, J.S Reid, K. Mulligan, N.S. Hellerstein, and C.H. Shackleton. 1991. Measurement of de novo hepatic lipogenesis in humans using stable isotopes. J. Clin. Invest. 87:1841-1852.

18. Wolfe, R.R., J.H. Shaw, and M.J. Durkot. 1985. Effect of sepsis on VLDL kinetics: responses in basal state and during glucose infusion. Am. J. Physiol. 248:E732-E740.

19. Hellerstein, M.K., and R.K. Neese. 1992. Mass isotopomer distribution analysis: a technique for measuring biosynthesis and turnover of polymers. Am. J. Physiol. 263:E988-E1001.

20. Kelleher, J.K., and T.M. Masterson. 1992. Model equations for condensation biosynthesis using stable isotopes and radioisotopes. Am. J. Physiol. 262: E118-E125.

21. Lee, P.W.N., S. Bassilian, Z. Guo, D. Schoeller, J. Edmond, A.E. Bergner, and L.O. Byerley. 1994. Measurement of fractional lipid synthesis using deuterated water $\left({ }^{2} \mathrm{H}_{2} \mathrm{O}\right)$ and mass isotopomer analysis. Am. J. Physiol. 266: E372-E383.

22. Wolfe, R.R., T.F. O'Donnell, M.D. Stone, D.A. Richmand, and J.F. Burke. 1980. Investigation of factors determining the optimal glucose infusion rate in total parenteral nutrition. Metab. Clin. Exp. 29:892-900.

23. Wolfe, R.R., J.R. Allsop, and J.F. Burke. 1979. Glucose metabolism in man: responses to intravenous glucose infusion. Metab. Clin. Exp. 28:210-220.

24. Grundy, S.M., H.Y.I. Mok, L. Zech, and D. Steinberg. 1979. Transport of very low density lipoprotein triglycerides in varying degrees of obesity and hypertriglyceridemia. J. Clin. Invest. 63:1274-1283.

25. McGuire, E.A.H., J.H. Helderman, J.D. Tobin, R. Andres, and M. Berman. 1976. Effects of arterial versus venous sampling on analysis of glucose kinetics in man. J. Appl. Physiol. 41:565-573.

26. Gotto, A.M., H.J. Pownall, and R.J. Havel. 1986. Introduction to the plasma lipoproteins. In Methods in Enzymology. Plasma Lipoproteins. J.P. Segrest and J.J. Albers, editors. Academic Press, Orlando. 3-40.

27. Havel, R.J., H.A. Eder, and J.H. Bragdon. 1955. The distribution and chemical composition of ultracentrifugally separated lipoproteins in humans. $J$. Clin. Invest. 34:1345-1353.

28. McGowman, M.W., J.D. Artiss, D.R. Strandbergh, and B. Zak. 1983. A peroxidase-coupled method for the colorimetric determination of serum triglycerides. Clin. Chem. 29:538-542.

29. Wolfe, R.R., and E.J. Peters. 1987. Lipolytic response to glucose infusion in human subjects. Am. J. Physiol. 252:E218-E223.

30. Wolfe, R.R. 1992. Radioactive and stable isotope tracers in biomedicine. In Principles and Practice of Kinetic Analysis. Wiley-Liss, New York.

31. Dixon, M., and E.L. Webb. 1979. Enzymes. In Enzymes. Academic Press, New York. 842-843.

32. Newsholme, E.A., and K. Taylor. 1969. Glycerol kinase activities in muscle from vertebrates and invertebrates. Biochem. J. 112:465-474.

33. Wasserman, D.H., D.B. Lacy, R.E. Goldstein, P.E. Williams, and A.P. Cherrington. 1989. Exercise-induced fall in insulin and increase in fat metabolism during prolonged muscular work. Diabetes. 38:484-491.

34. Hellerstein, M.K., C. Kletke, S. Kaempfer, K. Wu, and C.H. Shackleton. 1991. Use of mass isotopomer distributions in secreted lipids to sample lipogenic acetyl-CoA pool in vivo in humans. Am. J. Physiol. 261:E479-E486.

35. Chinkes, D., A. Aarsland, J. Rosenblatt, and R.R. Wolfe. 1996. A comparison of mass isotopomer dilution methods used to compute production of VLDL fatty acids in vivo in human subjects. Am. J. Physiol. 271:E373-E383.

36. Foster, D.M., P.H. Barrett, G. Toffolo, W.F. Beltz, and C. Cobelli. 1993. Estimating the fractional synthetic rate of plasma apolipoproteins and lipids from stable isotope data. J. Lipid Res. 34:2193-2205.

37. Dagher, F.J., J.H. Lyons, D.C. Finlayson, J. Shamsai, and F.D. Moore. 1965. Blood volume measurement: a critical study. Adv. Surg. 1:69-109.

38. Cook, H.W. 1991. Fatty acid desaturation and chain elongation in eucaryotes. In Biochemistry of Lipids, Lipoproteins and Membranes. D.E. Vance and J. Vance, editors. Elsevier Science Publishers, Amsterdam. 141-169.

39. Ajie, H.O., M.J. Connor, W.N.P Lee, S. Bassilian, E.A. Bergner, and L.O. Byerley. 1995. In vivo study of the biosynthesis of long-chain fatty acids using deuterated water. Am. J. Physiol. 269:E247-E252.

40. Gibbons, G.F., and D. Wiggins. 1995. Intracellular triacylglycerol lipase: its role in the assembly of hepatic very-low-density lipoprotein (VLDL). $A d v$. Enzyme Regul. 35:179-198.

41. Steiner, G., and M.F. Reardon. 1983. A new model of human VLDL metabolism based on simultaneous studies of its Apo B and triglyceride. Metab. Clin. Rev. 32:342-347.

42. Grundy, S.M., H.Y.I. Mok, L. Zech, and M. Berman. 1981. Influence of nicotinic acid on metabolism of cholesterol and triglycerides in man. J. Lipid Res. 22:24-36. 
43. Kissebah, A.H., S. Alfarsi, D.J. Evans, and P.W. Adams. 1982. Integrated regulation of very low density lipoprotein triglyceride and apolipoprotein-B kinetics in non-insulin-dependent diabetes mellitus. Diabetes. 31:217225 .

44. Boberg, J., L.A. Carlson, and U. Freyschuss. 1972. Determination of splanchnic secretion rate of plasma triglycerides and of total and splanchnic turnover of plasma free fatty acids in man. Eur. J. Clin. Invest. 2:123-132.

45. Kissebah, A.H., S. Alfarsi, and P.W. Adams. 1981. Integrated regulation of very low density lipoprotein triglyceride and apolipoprotein-B kinetics in man: normolipidemic subjects, familial hypertriglyceridemia and familial combined hyperlipidemia. Metab. Clin. Exp. 30:856-868.

46. Harris, W.S., W.E. Connor, D.R. Illingworth, D.W. Rothrock, and D.M. Foster. 1990. Effects of fish oil on VLDL triglyceride kinetics in humans. $J$. Lipid. Res. 31:1549-1558.

47. Cryer, P.E. 1994. Glucose counterregulation: prevention and correction of hypoglycemia in humans. Am. J. Physiol. 264:E149-E155.

48. Radziuk, J., and R. Inculet. 1983. The effect of ingested and intravenous glucose on forearm uptake of glucose and glucogenic substrate in normal man. Diabetes. 32:977-981.

49. DeFronzo, R.A. 1988. The triumvirate: b-cell, muscle, liver. A collusion responsible for NIDDM. Diabetes. 37:667-687.

50. Taskinen, M.-R., C.J. Packard, and J. Shepherd. 1990. Effect of insulin therapy on metabolic fate of apolipoprotein B-containing lipoproteins in NIDDM. Diabetes. 39:1017-1027.
51. Durrington, P.N., R.S. Newton, D.B. Weinstein, and D. Steinberg. 1982. Effect of insulin and glucose on very low density lipoprotein triglyceride secretion by cultured rat hepatocytes. J. Clin. Invest. 70:63-73.

52. Vogelberg, K.H., F.A. Gries, and D. Moschinski. 1980. Hepatic production of VLDL-triglycerides. Dependence of portal substrate and insulin concentration. Horm. Metab. Res. 12:688-694.

53. Lewis, G.F, K.D. Uffelman, L.W. Szeto, and G. Steiner. 1993. Effects of acute hyperinsulinemia on VLDL triglyceride and VLDL apoB production in normal weight and obese individuals. Diabetes. 42:833-842.

54. Duerden, J.M., S.M. Bartlett, and G.F. Gibbons. 1989. Long-term maintenance of high rates of very-low-density-lipoprotein secretion in hepatocyte cultures. A model for studying the direct effects of insulin and insulin deficiency in vitro. Biochem. J. 263:937-943.

55. Reaven, G.M. 1993. Role of insulin resistance in human disease (syndrome X): an expanded definition. Annu. Rev. Med. 44:121-131.

56. Fukuda, N., and J.A. Ontko. 1984. Interactions between fatty acid synthesis, oxidation, and esterification in the production of triglyceride-rich lipoproteins by the liver. J. Lipid Res. 25:831-842.

57. Gibbons, G.F., and F.J. Burnham. 1991. Effect of nutritional state on the utilization of fatty acids for hepatic triacylglycerol synthesis and secretion as very-low-density lipoprotein. Biochem. J. 275:87-92.

58. Baba, H., X.J. Zhang, K.R. Kunkel, and R.R. Wolfe. 1994. Effect of hepatectomy on glucose metabolism in the dog. Metab. Clin. Exp. 43:670-676. 